\title{
Can galaxy growth be sustained through HI-rich minor mergers?
}

\author{
M. D. Lehnert ${ }^{1,2}$, W. van Driel ${ }^{3,4}$, and R. Minchin ${ }^{5}$ \\ 1 Sorbonne Universités, UPMC Université Paris VI, Institut d'Astrophysique de Paris, 75014 Paris, France \\ 2 CNRS, UMR 7095, Institut d'Astrophysique de Paris, 98bis boulevard Arago, 75014 Paris, France \\ 3 GEPI, Observatoire de Paris, UMR 8111, CNRS, Université Paris Diderot, 5 place Jules Janssen, 92190 Meudon, France \\ e-mail: wim.vandriel@obspm.fr \\ ${ }^{4}$ Station de Radioastronomie de Nançay, Observatoire de Paris, CNRS/INSU USR 704, Université d'Orléans OSUC, \\ route de Souesmes, 18330 Nançay, France \\ 5 Arecibo Observatory, National Astronomy and Ionosphere Center, Arecibo, PR 00612, USA
}

Received 26 January 2015 / Accepted 10 October 2015

\begin{abstract}
Local galaxies with specific star-formation rates (star-formation rate per unit mass; $s S F R \sim 0.2-10 \mathrm{Gyr}^{-1}$ ) that are as high as distant galaxies $(z \approx 1-3)$, are very rich in HI. Those with low stellar masses, $M_{\star}=10^{8-9} M_{\odot}$, for example, have $M_{\mathrm{HI}} / M_{\star} \approx 5-30$. Using continuity arguments, whereby the specific merger rate is hypothesized to be proportional to the specific star-formation rate, along with HI gas mass measurements for local galaxies with high SSFR, we estimate that moderate-mass galaxies, $M_{\star}=10^{9-10.5} M_{\odot}$, can acquire enough gas through minor mergers (stellar mass ratios $\sim 4-100$ ) to sustain their star formation rates at $z \sim 2$. The relative fraction of the gas accreted through minor mergers declines with increasing stellar mass, and for the most massive galaxies considered, $M_{\star}=10^{10.5-11} M_{\odot}$, this accretion rate is insufficient to sustain their star formation. We checked our minor merger hypothesis at $z=0$ using the same methodology, but now with relations for local normal galaxies, and find that minor mergers cannot account for their specific growth rates, in agreement with observations of HI-rich satellites around nearby spirals. We discuss a number of attractive features, such as a natural downsizing effect, in using minor mergers with extended HI disks to support star formation at high redshift. The answer to the question posed by the title, "Can galaxy growth be sustained through HI-rich minor mergers?", is "maybe", but only for relatively low-mass galaxies and at high redshift.
\end{abstract}

Key words. galaxies: high-redshift - galaxies: formation - galaxies: evolution - galaxies: kinematics and dynamics - galaxies: ISM

\section{Introduction}

The importance of merging in the growth of galaxies is widely recognized. However, within the past decade, simulations have indicated that cosmological accretion of gas through the filamentary structures that develop as the Universe expands also played a significant, perhaps dominant role in supplying gas to galaxies (Kereš et al. 2005). The physical processes that may regulate the accretion of gas into the halos and onto galaxies proper have not been understood well (e.g., Mitchell et al. 2016). All gas accretion is driven by the evolution of the underlying dark matter and whether it is cosmological gas accretion or merging depends on how strongly the dark matter and baryons are coupled as they are accreted (Danovich et al. 2015).

In a stimulating analysis developed to constrain the growth of galaxies over cosmic time, Peng et al. (2014) assume a continuity in the stellar mass evolution of galaxies. Using the constancy of the faint-end slope of the galaxy mass function (e.g., Ilbert et al. 2013) and the (slightly) negative slope of the specific star-formation rate-stellar-mass $\left(s S F R-M_{\star}\right)$ relationship with redshift, they argue that minor mergers (stellar mass ratios 4-10) must be important for the stellar mass growth of galaxies. Allowing galaxies to accrete stellar mass as they grow owing to merging with smaller galaxies naturally restricts the evolution of the faint-end slope of the stellar mass function as the overall stellar mass density increases and gives rise to a negative slope in the $s S F R-M_{\star}$ relationship because not all stellar growth occurs via in situ star formation. Adopting a logarithmic slope of -0.1 for the $s S F R-M_{\star}$ relation and a faint end slope of -1.4 , Peng et al. (2014) determined that the specific merger (stellar) mass accretion rate as a function of the satellite mass, $s M M R=0.1 s S F R$. In other words, about $10 \%$ of the specific stellar growth per unit time of a galaxy is due to accreting stars through mergers, in addition to the usual definition of the sSFR as the specific growth rate due to ongoing star formation. They estimate that the range in stellar growth through mergers is about \pm 0.5 dex. They emphasized that this model applies only to galaxies with masses below the break in the mass function $\left(\sim 10^{10.7} M_{\odot}\right.$ which does not evolve significantly with redshift; Ilbert et al. 2013). The merger rate then becomes $R\left(M_{\mathrm{sat}}\right)=x \cdot \operatorname{sMMR}\left(M_{\star, \mathrm{sat}}\right)=0.1 \cdot x \cdot s S F R$, where $x$ is the ratio of the stellar mass of the central galaxy, $M_{\star, \text { cen }}$, to the satellite, $M_{\star, \text { sat }}$.

Having characterized the merger rate in this way, if we include the gas content of the merging galaxies, in particular their HI content using the ratio, $M_{\mathrm{HI}} / M_{\star}$, how much mass would then be accreted for given stellar accretion rates? These are the goals of the paper: to estimate the gas accretion rate for gas-rich minor mergers and compare it to the amount of gas necessary to sustain galaxy growth at $z \approx 2$ and 0 .

\section{Methodology}

Our methodology relies on two strong assumptions. The first is that the relative HI gas content (i.e., the $M_{\mathrm{HI}} / M_{\star}$ ratio) of galaxies at $z \sim 2$ is the same as for our local sample with extremely high sSFR, which we selected to have stellar masses and sSFR 
similar to those at $z \sim 1-3$ (Appendix A). This assumption implies that the relative $\mathrm{HI}$ gas content of galaxies is not a function of redshift, but that it increases with sSFR. Since direct observations of the HI content of distant galaxies are not currently feasible, to test this assumption we used CO line observations of molecular gas content as a proxy for their total gas content.

Our CO observations of our local high-sSFR galaxy sample suggest (Lehnert et al., in prep.) that they have molecular gas fractions, $f_{\text {gas }}=M_{\mathrm{H}_{2}} /\left(M_{\star}+M_{\mathrm{H}_{2}}\right)$, as high as those of galaxies at high redshift and far exceeding those of local galaxies with average sSFR (Tacconi et al. 2010). This comparison suggests that distant high-sSFR galaxies may also have substantial quantities of HI.

However, the similarity of molecular gas fractions between our low redshift sample and distant galaxies in itself is not evidence that the two populations are similar in their overall gas content. In fact, in the literature, there are many papers that suggest the co-moving HI content of the Universe may be constant with redshift or evolve only weakly (cf. Prochaska \& Wolfe 2009; Martin et al. 2010; Zafar et al. 2013). As we show in Sect. 4, a strongly evolving ratio of the HI to stellar mass ratio is consistent with a non-evolving co-moving density of HI. This is simply due to the fact that the co-moving stellar density evolves very strongly with redshift (Ilbert et al. 2013).

The second assumption is based on the continuity approach to estimate the growth of galaxies with cosmic time (Peng et al. 2014) - which simply states that galaxies must evolve continuously from one epoch to the next. Adopting their formalism for galaxy growth (see Introduction), we will assume that galaxies grow $10 \%$ of their stellar mass through minor mergers, and it implies that the merger probability increases linearly with merger mass ratio. Given the uncertainties inherent in estimating merger rates at high redshift (e.g., Bertone \& Conselice 2009; Bluck et al. 2012), it is not clear if this is what is observed. If the merger probability goes as the stellar mass ratio of the merger, then the mass acquired per logarithmic bin is constant. Peng et al. (2014) suggest this is consistent with merger rate estimates at high redshift, after a detailed comparison (at least for massive galaxies; e.g., Newman et al. 2012).

To estimate the amount of gas accreted through minor mergers using the formalism of Peng et al. (2014) requires an estimate of the gas to stellar mass ratio as a function of stellar mass. While measuring the total $\mathrm{H}_{2}$ mass of distant galaxies at $z=1-3$ is feasible, it is currently impossible to measure their HI content, which we can only estimate through models or by analogy with local objects that may be similar to distant galaxies. To this end, we conducted a program of sensitive, single dish observations of almost 200 nearby galaxies with extremely high sSFR, meaning as high as observed at $z=1-3$ (Elbaz et al. 2007; Daddi et al. 2007). We describe the selection process of these galaxies and the HI observations and results in Appendix A.

\section{Gas accretion through minor mergers}

Assuming that the relative HI content of galaxies at $z \sim 2$ is the same as that of local galaxies with similarly high sSFR, we can estimate the stellar plus gas accretion rate for high redshift galaxies. To do this, we integrate the merger rate, $R\left(M_{\mathrm{sat}}\right)$, multiplied by the total baryonic mass, $M_{\star}+M_{\mathrm{HI}}+M_{\mathrm{H}_{2}}$, over the range 1 to $x_{\text {lim }}$, where $x_{\text {lim }}$ spans the entire range of 1 to 100 . We calculate these integrals as a function of $x_{\text {lim }}$ for a few fixed values of $M_{\text {cen }}\left(\log M_{\star}\left(M_{\odot}\right)=9.0,9.5,10.0,10.5\right)$ and have fixed the total stellar mass accretion rate at $0.1 \cdot s S F R$ for all ranges of the integration with respect to $x$ (Fig. 1). To set the

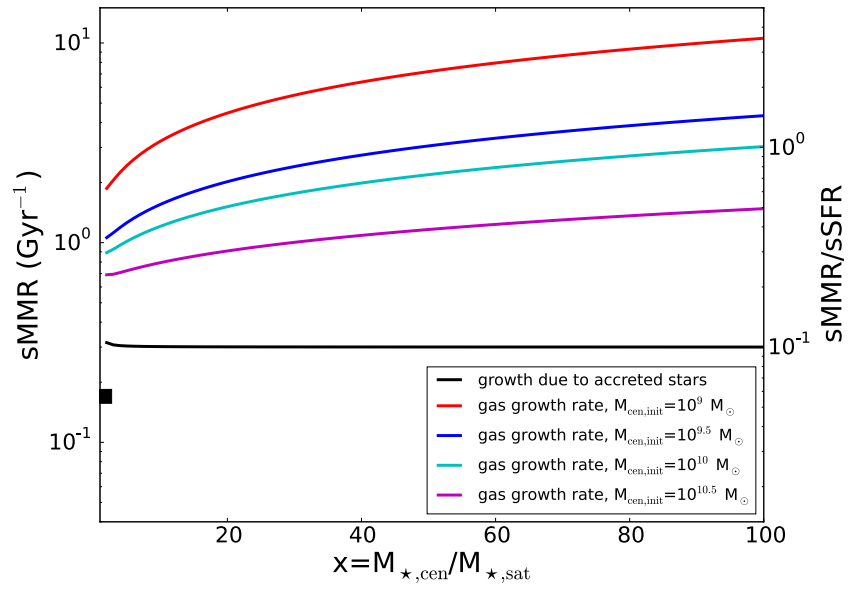

Fig. 1. Specific merger mass accretion rate (in units of $\mathrm{Gyr}^{-1}$ ) as a function of the stellar mass ratio of mergers, $x$, for $z=2$. The lines represent an SMMR fixed at $10 \%$ of the SSFR due to accreted stars (black solid line) and the total sMMR (including stars and gas) for various initial central galaxy stellar masses (indicated in the legend). We assumed $S S F R=3 \mathrm{Gyr}^{-1}$. On the righthand ordinate, we indicate the $S S M R / S S F R$ ratio to compare the stellar mass growth due to merging and to star formation. The black point indicates the merger rate determined for $z \approx 2-4$ by Tasca et al. (2014) for an assumed merger time scale of $1 \mathrm{Gyr}$ - the value indicated is likely a lower limit since the time scale may be shorter.

scale, we assumed a constant $S S F R=3 \mathrm{Gyr}^{-1}$, which is approximately the value measured at $z \sim 2$ (Daddi et al. 2007) and similar to our sample of nearby high-sSFR galaxies with measured HI masses. For the contribution of the HI gas, we used the relationship $\log M_{\mathrm{HI}} / M_{\star}=-0.59 \log M_{\star}+5.9$ from our HI survey of local galaxies with high sSFR (Appendix A), which has been measured for objects with $8.0 \leq \log M_{\star} \leq 10.8$, or approximately over 0.002 to 1.2 times the break mass in the stellar mass function (Ilbert et al. 2013). As already emphasized by Peng et al. (2014), this is the mass range where the stellar mass accretion model is appropriate.

For the contribution from $\mathrm{H}_{2}$ we simply assumed a gas fraction of 0.5 , consistent with $\mathrm{CO}$ observations of galaxies at $z \approx 2$ (Daddi et al. 2010; Aravena et al. 2010; Tacconi et al. 2013) and of our sample of nearby galaxies with comparably high sSFR and stellar masses (Lehnert et al., in prep.). We note however that the exact value of the molecular gas fraction assumed does not change the result significantly as the $\mathrm{HI}$ is the dominant contributor to the gas accretion rate for the mass range probed in this analysis. If the molecular gas fraction increases with decreasing stellar mass (e.g., Erb et al. 2006; Daddi et al. 2010; Tacconi et al. 2010) then the gas accretion rate through minor mergers would increase. Our analysis is conservative in this respect.

Figure 1 shows the total specific merger mass accretion over a broad range of merger stellar mass ratios. The stellar mass accretion rates assumed are consistent with observations (Tasca et al. 2014), although we note that this comparison assumes a merger time of a Gyr, while it is likely lower. We see that including the gas implies that for galaxies with relatively low initial stellar masses $\left(M_{\text {cen }}<10^{10.5} M_{\odot}\right)$, the specific gas accretion rate through minor mergers, with $x \gtrsim 20-50$, is sufficient to fuel the specific growth rate of galaxies (as $s M M R / s S F R \gtrsim 1$ ). For high-mass galaxies, near the break in the mass distribution function, even minor mergers with relatively large mass ratios, up to $x=100$, are insufficient to fully supply the necessary gas 


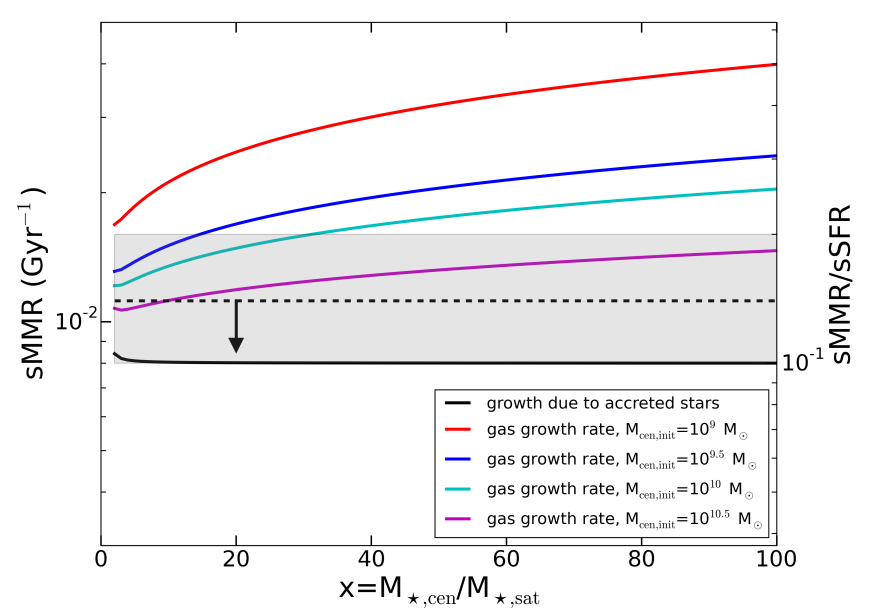

Fig. 2. Specific merger mass accretion rate (in units of $\mathrm{Gyr}^{-1}$ ) as a function of the stellar mass ratio of mergers, for $z=0$. The lines are exactly the same as in Fig. 1 but now we use the measured mean HIto-stellar mass ratio as a function of stellar mass from a reference sample of local galaxies with normal sSFR from Papastergis et al. (2012), and $S S F R=0.08 \mathrm{Gyr}^{-1}$. We also indicate the determinations of the HI accretion rate from two surveys of local spirals (Sancisi et al. 2008; Di Teodoro \& Fraternali 2014, gray-shaded region and dashed lines respectively; see text for details).

to support the relative growth of galaxies. We further note including even lower mass satellite galaxies (higher $x$ ) will not increase the gas accretion rate substantially as although their HI gas fractions are high, their total HI masses are small - the effect saturates.

\subsection{Constraints from local galaxies}

Given that we do not have good constraints on the merger rate at high redshifts, especially at low stellar mass ratios, and on the dependence of the merger timescale on the mass ratio, it is important to test the minor merger hypothesis locally. Analyses of observations of $\mathrm{HI}$ in low-mass galaxies interacting/merging with nearby star-forming galaxies suggest that minor merger accretion rates of $0.1-0.3 M_{\odot} \mathrm{yr}^{-1}$ (Sancisi et al. 2008; Di Teodoro \& Fraternali 2014).

Using our model, but now assuming the measured mean relationship between the HI mass and stellar mass for a reference sample of local galaxies with typical sSFR $\left(\log M_{\mathrm{HI}} / M_{\star}=-0.43\right.$ $\log M_{\star}+3.75$; Papastergis et al. 2012) and a molecular gas fraction of $10 \%$, we again estimate the stellar plus gas accretion rates as a function of stellar mass of the central galaxy and the merger mass ratio, $x$ (Fig. 2). We see that, in the local Universe, minor mergers are far from being able to supply the gas necessary to support the relative growth of galaxies, and this, unlike at high redshift, even at the lowest initial central galaxy stellar masses and for a limiting $x=100$.

Our predictions can be compared to the HI accretion rates determined for local galaxies (Sancisi et al. 2008; Di Teodoro \& Fraternali 2014), and are found to be in general agreement. In the sample of Di Teodoro \& Fraternali (2014), the average stellar mass is $10^{10.6} M_{\odot}$ and their upper limit to the HI mass accretion rate of $0.28 M_{\odot} \mathrm{yr}^{-1}$ implies $s M M R=$ $0.007 \mathrm{Gyr}^{-1}$. However, it is not clear what the relative stellar masses of their minor mergers are, but the baryonic mass ratios (stellar plus HI mass) are $M_{\text {bar,cen }} / M_{\text {bar,sat }} \gtrsim 5$. It is likely that the stellar mass ratio is higher, since the satellites are richer in $\mathrm{HI}$ than the central, more massive galaxy. In addition, the average
SSFR of their galaxies is less than what we assumed in our analysis. We have therefore scaled up their sSFR by a factor of 1.6 for the purpose of this comparison, to make it consistent with our assumptions about the proportionality of the minor merger rate with the sSFR. Comparing with the results from Sancisi et al. (2008) is more difficult due to a relative lack of specific information about their sample and estimates. They find an accretion rate of $0.1-0.2 M_{\odot} \mathrm{yr}^{-1}$ for galaxies with a star formation rate of $\approx 1 M_{\odot} \mathrm{yr}^{-1}$ which implies an $s M M R / s S F R$ of $0.1-0.2$. The latter is what we compare with in Fig. 2, not the sMMR directly.

We note that individual galaxies in our sample have uncertainties of about $30 \%$ in their estimated HI to stellar mass ratios and that the binned mass ratio data of the reference sample from Papastergis et al. (2012) typically have about the same uncertainties (see Appendix A). The uncertainty in the zero-point of the fit for the high sSFR galaxies in the $\log M_{\mathrm{HI}} / M_{\star}-\log M_{\star}$ plane is 0.3 dex. If we take this into account, analyzing it as a possible systematic uncertainty, implies that the estimates of the sMMR as a function of $x$ have a systematic range of about $\pm 35 \%$. As we do not have an estimate of the uncertainties in the fits estimated in Papastergis et al. (2012), we will assume they are similar. Such a level of uncertainty would not change our general conclusions of whether minor mergers play a role at all and over what redshift range, although it would certainly change our detailed conclusions, namely at what stellar masses are minor mergers likely to play a role in supporting ongoing star formation.

\subsection{Constraints from co-moving density of $\mathrm{HI}$}

A basic test of whether distant galaxies are more HI-rich than local galaxies, as we have assumed, is to estimate the co-moving density of HI. To do this, we multiply the stellar mass function of galaxies by the relationship between the HI and stellar masses used in deriving the specific merger gas accretion rate for distant and local galaxies. Multiplying our best fit to the local galaxies with high sSFR (Appendix A) by the stellar mass function at $z=2.0-2.5$, only considering the star forming galaxies which dominate the mass budget (Ilbert et al. 2013), we find that the co-moving density of HI calculated in this way is only a fraction, $20 \%$, of the total HI co-moving density estimated from sub- and damped Lyman- $\alpha$ absorbers (sub-DLAs and -DLAs respectively; Zafar et al. 2013). The uncertainty in this estimate is large, at least a factor of 2 . If anything, since absorption line studies probe gas that may not always be directly associated with the extended gas disks of galaxies, we expect that using this method we will underestimate the total co-moving HI density. Furthermore, there are additional reasons to not expect estimating the total co-moving mass of $\mathrm{HI}$ as we have done to agree with absorption line studies. HI absorption lines probe gas whose characteristics are likely different from emission line gas (spin temperatures, dynamical state, fraction of warm to cold gas, etc.) beyond whether the gas is predominately associated with galaxy disks as we are assuming. We only intended this comparison to gauge whether we are likely to over-estimate the HI content of distant galaxies, and observations suggest we are not.

\section{Discussion}

Our analysis indicates that accreting gas through gas-rich minor mergers provides a sufficient reservoir of gas to sustain the high specific star formation rates observed for galaxies of relatively low mass (below about $\left.M^{\star}\right)$ at high redshifts $(z \sim 2)$. There are 
several advantages to this hypothesis: (1) it may mimic cosmological accretion; (2) it will lead to shorter merger times than if we consider only their stellar masses; (3) it will not thicken disks substantially; (4) it may lead to relatively metal-rich galactic outskirts; and (5) it is consistent with weak or no evolution in the co-moving density of HI. We very briefly discuss each of these possible advantages.

HI-rich minor mergers may mimic cosmological gas accretion: if the HI disks at high redshift are akin to their low redshift counterparts, they will be rotationally supported and dynamically cold, especially in their outer regions (velocity dispersions, $\sigma_{\mathrm{HI}} \sim 6-10 \mathrm{~km} \mathrm{~s}^{-1}$ and $v_{\text {rot }} / \sigma_{\mathrm{HI}}=3-10$; Stilp et al. $2013 \mathrm{~b}, \mathrm{a})$. In addition, the warm neutral gas has a very short turbulent dissipation timescale, of-order $10 \mathrm{Myr}$, meaning, if disturbed, it will dissipate energy injected as turbulence quickly in a dynamical time or less even for low-mass galaxies. In several important ways, the HI gas in extended disks would behave similarly to cold streams that have penetrated deeply into the halo (Danovich et al. 2015). The small merging galaxies would be accreted along filaments, their HI gas is cold and stable, and they have high angular momentum both from rotation and due to their orbits. In fact, the extended HI disks may induce a cooling wake in the hotter halo gas as the galaxy falls deeper into the halo potential allowing more gas to be accreted beyond that provided directly by the merger (Marasco et al. 2012) - this would significantly increase the impact of direct gas accretion through minor mergers.

$H_{I-r i c h}$ minor mergers will have relatively short(er) merging times: in gas-rich mergers the dominating, dynamically cold gaseous component would substantially decrease the merger time scale (Lotz et al. 2008; Hopkins et al. 2010). Galaxies with stellar masses of $10^{8-9} M_{\odot}$ have approximately 10 times as much of their mass in HI as in stars. If regarded as truly minor mergers at 1:100, considering only their stellar masses, they are actually $\sim 1: 10$ mergers considering their gas content and will have a substantially reduced merger timescale (by a factor of 2 or more; Hopkins et al. 2010).

HI-rich minor mergers will not thicken galactic disks substantially: minor mergers, especially frequent ones as advocated in this analysis, might substantially heat the disk and result in thick(er) disks. However, if the gas fractions are high in the merging satellite, its impact on the thickening of the disk is relatively small (Hopkins et al. 2008). While we do not show the heating rate of the galaxies in our simple model, the impact on the vertical heating rate of minor mergers is proportional to $1-f_{\text {gas,total }}$, the ratio of the total gas mass $\left(\mathrm{HI}+\mathrm{H}_{2}+\mathrm{He}\right)$ and the total mass (gas + stars) of the galaxy. Since the majority of minor mergers are gas rich, $f_{\text {gas,total }} \gg 0.9$, their impact on the heating the disk of the central galaxy will be small (Hopkins et al. 2008).

HI-rich minor mergers may lead to relatively metal-rich galactic outskirts: minor mergers may also allow for metal enriched gas to be accreted. Observations of HII regions in the outskirts of nearby galaxies suggest that they have attained substantial metal enrichment (Bresolin et al. 2012) which cannot be due to the currently observed levels of star formation, even if sustained for a Hubble time (Bresolin et al. 2012). While not demanding it, these results suggest that gas is accreted already enriched, as would be the case for low-mass satellite galaxies.

HI may be the conveyor belt sustaining the growth of galaxies and growing galaxies through gas-rich minor mergers is consistent with no evolution in the co-moving density of $\mathrm{H}$ : this idea means that HI is the ultimate gas reservoir which, through its own collapse or conversion to molecular gas (a conveyor), sustains the long-term star formation and thus the growth of galaxies. If true, we would expect that as the stellar mass content of the Universe increased, the HI to stellar mass ratio would decrease roughly in lock step, and thus the total HI content of the Universe would remain roughly constant. To test whether our scaling of the HI to stellar mass ratios of galaxies in the local and distant, $z \approx 2$, Universe, is consistent with this conveyor belt hypothesis, we need to estimate $\Omega_{\mathrm{HI}}$, the co-moving density of HI relative to the closure density. $\Omega_{\mathrm{HI}}$ is constant over the redshift range $1-5$, and maybe even down to $z=0$ (Zafar et al. 2013; Martin et al. 2010), while the star formation rate density has declined significantly and the co-moving stellar mass density of the Universe has increased (Ilbert et al. 2013). Although we do not account for all of the HI density estimated from sub- and DLAs at high redshift (Zafar et al. 2013) in our analysis, only $\sim 1 / 5$ (Sect. 3.2), we can estimate the evolution of the co-moving density of HI implied by our analysis to check for consistency with this hypothesis.

Is our analysis therefore consistent with a constant amount of Hi per unit co-moving volume, supporting the hypothesis that $\mathrm{HI}$ is the reservoir out of which star formation is sustained? For this estimate, we assume the mass functions given in Ilbert et al. (2013) for star forming galaxies at $z=2-2.5$ and $z=0.2-0.5$. While they do not estimate the stellar mass distribution function at $z=0$, the ultimate evolution of the total HI content is likely small (Zafar et al. 2013) as is the change in stellar mass density. Doing this, using our ratio for HI to stellar masses as a function of stellar mass (Appendix A) for $z=2-2.5$ and that from Papastergis et al. (2012) for galaxies at $z=0$, we find that the ratio of HI densities, $\rho_{\mathrm{HI}}$, at $z \sim 2$ and $z \sim 0$ is about 1.5 with an uncertainty of about a factor of $\sim 3$. Even though the estimate is crude, it does suggest that overall, our analysis is consistent with no evolution in the co-moving density of HI and implies that HI gas is the reservoir out of which galaxies move up the stellar mass hierarchy.

In this picture, this reservoir may be supplied through minor mergers, at least at high redshifts and for relatively low-mass galaxies. Furthermore, downsizing is a natural result of galaxies becoming increasingly gas starved as they grow in mass and, overall, as their redshift decreases.

Acknowledgements. We wish to thank the anonymous referee for carefully reading our manuscript and making a number of extremely useful comments. We wish to also thank Paola Di Matteo for her comments on and enthusiasm for this work. The Nançay Radio Astronomy Station is operated as part of the Paris Observatory, in association with the Centre National de la Recherche Scientifique (CNRS) and partially supported by the Région Centre in France. The Arecibo Observatory is operated by SRI International under a cooperative agreement with the National Science Foundation (AST-1100968), and in alliance with Ana G. Méndez-Universidad Metropolitana, and the Universities Space Research Association. This research has made use of the HyperLeda database (http: //leda. univ-lyon1.fr), the NASA/IPAC Extragalactic Database (NED) which is operated by the Jet Propulsion Laboratory, California Institute of Technology, under contract with the National Aeronautics and Space Administration and the Sloan Digital Sky Survey which is managed by the Astrophysical Research Consortium for the Participating Institutions.

\section{References}

Aravena, M., Carilli, C., Daddi, E., et al. 2010, ApJ, 718, 177 Bertone, S., \& Conselice, C. J. 2009, MNRAS, 396, 2345 Bluck, A. F. L., Conselice, C. J., Buitrago, F., et al. 2012, ApJ, 747, 34 Bresolin, F., Kennicutt, R. C., \& Ryan-Weber, E. 2012, ApJ, 750, 122 Brinchmann, J., Charlot, S., White, S. D. M., et al. 2004, MNRAS, 351, 1151 Daddi, E., Dickinson, M., Morrison, G., et al. 2007, ApJ, 670, 156 Daddi, E., Bournaud, F., Walter, F., et al. 2010, ApJ, 713, 686 Danovich, M., Dekel, A., Hahn, O., Ceverino, D., \& Primack, J. 2015, MNRAS, 449, 2087 
M. D. Lehnert et al.: Growth through HI-rich minor mergers

Di Teodoro, E. M., \& Fraternali, F. 2014, A\&A, 567, A68

Drory, N., Bender, R., \& Hopp, U. 2004, ApJ, 616, L103

Elbaz, D., Daddi, E., Le Borgne, D., et al. 2007, A\&A, 468, 33

Erb, D. K., Steidel, C. C., Shapley, A. E., et al. 2006, ApJ, 646, 107

Haynes, M. P., Giovanelli, R., Martin, A. M., et al. 2011, AJ, 142, 170

Hopkins, P. F., Hernquist, L., Cox, T. J., Younger, J. D., \& Besla, G. 2008, ApJ, 688,757

Hopkins, P. F., Croton, D., Bundy, K., et al. 2010, ApJ, 724, 915

Huang, S., Haynes, M. P., Giovanelli, R., et al. 2012, AJ, 143, 133

Ilbert, O., McCracken, H. J., Le Fèvre, O., et al. 2013, A\&A, 556, A55

Isobe, T., Feigelson, E. D., \& Nelson, P. I. 1986, ApJ, 306, 490

Kauffmann, G., Heckman, T. M., White, S. D. M., et al. 2003, MNRAS, 341, 54

Kereš, D., Katz, N., Weinberg, D. H., \& Davé, R. 2005, MNRAS, 363, 2

Lotz, J. M., Jonsson, P., Cox, T. J., \& Primack, J. R. 2008, MNRAS, 391, 1137

Marasco, A., Fraternali, F., \& Binney, J. J. 2012, MNRAS, 419, 1107

Martin, A. M., Papastergis, E., Giovanelli, R., et al. 2010, ApJ, 723, 1359

Mitchell, P. D., Lacey, C. G., Cole, S., \& Baugh, C. M. 2016, MNRAS, 456, 1459
Moustakas, J., Coil, A. L., Aird, J., et al. 2013, ApJ, 767, 50

Newman, A. B., Ellis, R. S., Bundy, K., \& Treu, T. 2012, ApJ, 746, 162

Papastergis, E., Cattaneo, A., Huang, S., Giovanelli, R., \& Haynes, M. P. 2012, ApJ, 759, 138

Peng, Y.-j., Lilly, S. J., Renzini, A., \& Carollo, M. 2014, ApJ, 790, 95

Prochaska, J. X., \& Wolfe, A. M. 2009, ApJ, 696, 1543

Saintonge, A. 2007, AJ, 133, 2087

Salim, S., Rich, R. M., Charlot, S., et al. 2007, ApJS, 173, 267

Sancisi, R., Fraternali, F., Oosterloo, T., \& van der Hulst, T. 2008, A\&ARv, 15, 189

Sorba, R., \& Sawicki, M. 2015, MNRAS, 452, 235

Stilp, A. M., Dalcanton, J. J., Skillman, E., et al. 2013a, ApJ, 773, 88

Stilp, A. M., Dalcanton, J. J., Warren, S. R., et al. 2013b, ApJ, 765, 136

Tacconi, L. J., Genzel, R., Neri, R., et al. 2010, Nature, 463, 781

Tacconi, L. J., Neri, R., Genzel, R., et al. 2013, ApJ, 768, 74

Tasca, L. A. M., Le Fèvre, O., López-Sanjuan, C., et al. 2014, A\&A, 565, A10

Tremonti, C. A., Heckman, T. M., Kauffmann, G., et al. 2004, ApJ, 613, 898

Zafar, T., Péroux, C., Popping, A., et al. 2013, A\&A, 556, A141 


\section{Appendix A: Selection of a local high-sSFR galaxy sample, $\mathrm{H}$ observations and results}

\section{A.1. Sample selection}

All galaxies were selected using the Sloan Digital Sky Survey (SDSS) data release DR7 ${ }^{1}$, in combination with certain physical parameters of the galaxies (stellar masses, star-formation rates, rest-frame optical colors, etc.) which were taken from the publicly available SDSS "value added" MPA/JHU catalogs (Brinchmann et al. 2004; Kauffmann et al. 2003; Salim et al. 2007; Tremonti et al. 2004). The consecutive steps in the selection process are as follows.

1. Redshift: $0.01<z<0.05$. We chose $z=0.01$ as our lower limit so that the SDSS spectrograph fiber subtends at least the central $200 \mathrm{pc}$ to give meaningful results (see Elbaz et al. 2007 , for this in comparison with distant galaxies). The upper limit of $z=0.05$ (or a radial velocity of $15000 \mathrm{~km} \mathrm{~s}^{-1}$ ) is the estimated limit for a good HI detection rate in our survey;

2. Color: rest-frame $(g-r)<0.63 \mathrm{mag}$. We selected only the bluest $25 \%$ of all galaxies in the MPA/JHU catalogs;

3. sSFR: highest $5 \%$ of galaxies with blue rest-frame colors. We fitted a line to the resulting main sequence in the $\log (s S F R)-$ $\log \left(M_{\star}\right)$ plane, which has a slightly negative slope, -0.2 . We then, as a function of stellar mass, selected the $5 \%$ of galaxies with the highest sSFRs. Although low-mass galaxies on average have a higher sSFR than the more massive ones, all objects in all $M_{\star}$ ranges span similar ranges in SSFR as the distant galaxies typically do: they have $S S F R \approx 0.2-$ $10 \mathrm{Gyr}^{-1}$, compared to $0.7 \mathrm{Gyr}^{-1}$ and $2 \mathrm{Gyr}^{-1}$, for average main sequence galaxies at $z \sim 1$ and $z \sim 2$, respectively (e.g., Elbaz et al. 2007; Daddi et al. 2007);

4. Total stellar mass: $M_{\star} \geq 10^{8} M_{\odot}$. We adopted this limit in order to avoid galaxies in the low-mass range where the SDSS is becoming highly incomplete. This lower limit in mass also (generously) ensures that our sample spans the entire stellar mass range of galaxies with well-determined sSFR at $z \sim 1-3$.

\section{A.2. Hi observations and results}

Our final high-sSFR local galaxy sample contains 2698 objects. Of those, we have 329 clear HI detections at our disposal for further analysis (104 from our Nançay and 23 from our Arecibo surveys, and 202 from the literature), as well as 7 marginal detections (6 Nançay and 1 Arecibo) and 39 non-detections (29 Nançay and 10 Arecibo). Publication of the full results is in preparation.

The Nançay Radio Telescope (NRT) is a 100 meter-class instrument. Its HPBW is 3.5 in right ascension, while in declination it is $23^{\prime}$ for $\delta<20^{\circ}$, increasing to $30^{\prime}$ at the northern limit of our survey. Observations were taken in position-switching mode, in the period January 2012-March 2013 for a total of $400 \mathrm{~h}$ of telescope time. The data were reduced using standard NRT routines, smoothing to a velocity resolution of $18 \mathrm{~km} \mathrm{~s}^{-1}$.

The Arecibo radio telescope has a $305 \mathrm{~m}$ diameter. The HPBW of the $L$-band wide (LBW) receiver used is 3'.1 $\times 3.5$. Observations were taken in position-switching mode, in the period January 2013-May 2014 for a total of 14 hours of telescope time.

\footnotetext{
1 http://wWw. sdss.org/dr7/
}

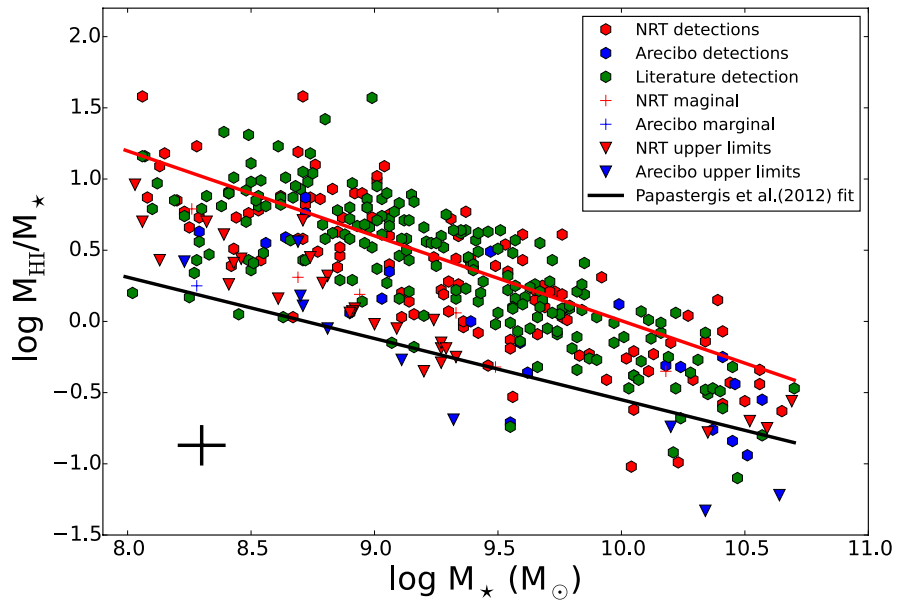

Fig. A.1. Logarithm of the HI to stellar mass ratio, $\log M_{\mathrm{HI}} / M_{\star}$, as a function of the logarithm of the stellar mass, $\log M_{\star}$ (in units of $M_{\odot}$ ) for the galaxies in our high sSFR sample. Detections with $S / N>5$ are shown as hexagons, marginal detections of S/N 3-5 are shown as crosses, and upper limits shown as downward pointing triangles. The colors indicate the telescope used, or if the detection are from the literature as indicated in the legend. We indicate the typical uncertainty in each quantity for individual galaxies by the black cross in the lower left corner of the plot (see text for details). The red line is the best fit using the Buckley-James method to fit censored data (i.e., including our upper limits, which gives $\log M_{\mathrm{HI}} / M_{\star}=-0.59 \log M_{\star}+5.9$; Isobe et al. 1986). The black line is the fit to the reference sample of local galaxies with typical sSFR from Papastergis et al. (2012).

The data were reduced using standard Arecibo Observatory IDL routines, smoothing to a velocity resolution of $20 \mathrm{~km} \mathrm{~s}^{-1}$.

We found published HI detections of a further 293 target galaxies, of which we used 202 for further analysis. They were found predominantly $(68 \%)$ from matching our SDSS coordinates within a search radius of $4^{\prime \prime}$ to those in the $\alpha .40$ catalog (Haynes et al. 2011) of the blind Arecibo ALFALFA survey, from which we used only the reliable quality 1 detections, which have a signal-to-noise ratio greater than 6.5 (see Saintonge 2007). We also searched the on-line NED and HyperLeda databases, within a radius of $15^{\prime \prime}$. However, after visual inspection of SDSS images, we noted that 54 detections are associated in the MPA/JHU catalog with SDSS spectroscopic sources which are actually sub-structures in the galaxies outside the nuclear region, such as star forming regions. As their associated SDSS photometric sources do not represent the entire galaxy, the estimated stellar masses given in the MPA/JHU cata$\log$ are therefore likely (severe) underestimates for the galaxy as a whole, and we did not use them further for our study. We also discarded 37 cases where one or more other galaxies within the radio telescope beam were likely to confuse the HI detection of the target.

\section{A.3. Uncertainties}

The uncertainties in the stellar mass estimates of our sample of galaxies were determined by taking the $1 \sigma$ confidence level of each galaxy from the MPA/JHU value added catalogs. To estimate the typical uncertainty for individual galaxies, we simply calculate the average of all uncertainties. The typical uncertainty is shown as a cross in Fig. A.1, showing each individual uncertainty would make the plot illegible. We find that the average uncertainty in the stellar mass estimates we 
used is 20\%. Papastergis et al. (2012) used a somewhat different way to estimate stellar masses of the reference sample they use to evaluate the gas-to-stellar mass ratio as function of $M_{\star}$ for local HI-detected galaxies with typical SSFR, see Huang et al. (2012) for details. As we do not have a simple way of estimating the systematic uncertainties between the two stellar mass estimation methods we will ignore this, but we note that in our experience different mass estimates usually agree within about 0.3 dex (e.g., Drory et al. 2004; Moustakas et al. 2013; Sorba \& Sawicki 2015). For both our NRT and Arecibo HI data, we find a typical relative uncertainty of about $15 \%$ and a systematic uncertainty of about $10 \%$, which we determined through regular observations of Hi line flux calibrator galaxies. To estimate the total uncertainty we added each uncertainty in quadrature. We have indicated the typical uncertainty in Fig. A.1 for both the stellar mass and the ratio of HI to stellar mass.

The uncertainties in our fit to the high sSFR galaxies are 0.03 in the slope and 0.3 in the zero-point. Thus, the latter will effect our conclusions the most significantly. Unfortunately, Papastergis et al. (2012) do not provide an estimate of the uncertainties in their fit. 